\title{
THROMBOELASTROMETRY AS A GUIDE FOR BLOOD MANAGEMENT IN THORACOABDOMINAL ANEURYSM SURGERY
}

\author{
Bueno L. ', Martins K. ', Beltrao R. ', Fossati S. ', Cernuda I. ${ }^{2}$, Aguilar C. ${ }^{1}$ \\ Department of Anaesthesiology and Critical Care ${ }^{1}$. Department of Vascular Surgery. ${ }^{2}$ \\ Hospital Clínlco San Carlos. Madrid.
}

BACKGROUND:

Rotational thromboelastometry (ROTEM ${ }^{\circledR}$ ) is a viscoelastometric method that tests coagulation in a dynamic and global way analysing a whole blood sample.

\section{GOAL OF THE STUDY:}

The aim of this study is to assess the results of using thromboelastometry as guidance for haemoderivates management in elective open repair of aortic aneurysm as an attempt to reduce postoperative complications associated to non-selective haemoderivates transfusion.

\section{MATERIAL AND METHODS:}

A prospective cohort study was performed with nonrandomized sampling using a retrospective control group.

29 patients undergoing thoracoabdominal aneurysms open repair were included.

Homogeneously distributed in $\mathbf{2}$ groups:

1. Group A (Control): 14 patients. Transfused according to classic protocols.

2. Group B (Test): 15 patients. Thromboelastometric based transfusion algorithms

\section{RESULTS AND DISCUSSION:}

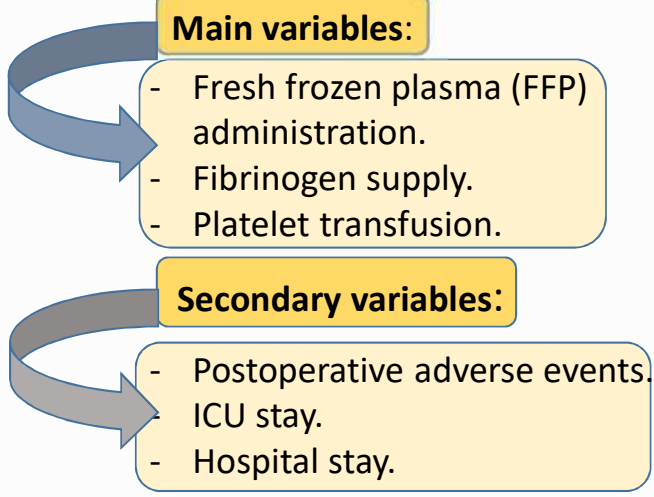

Decremental transfusion of fresh frozen plasma in group B with respect to group $A(p=0,017)$. (Image 1)

Increased use of fibrinogen, that was administrated in $86,7 \%$ of patients in group $B$, comparing to $35,7 \%$ patients in group $A(p=0,009)$. (Image 2)

A rise in the use of platelets in group A $(92,8 \%)$, comparing to group B $(73,3 \%)(p=0,038)$. (Image 3$)$

35.7\% patients in group A had postoperative respiratory complications, with respect to $6.7 \%$ in group $B$, although this difference were not statistically significant $(p=0,08)$. (Image 4).

$\square$ No differences were found in prothrombin complex administration, homologous blood transfusión, UCl or hospital stay.

Image 1. Fresh frozen plasma transfusion. $(p=0.017)$
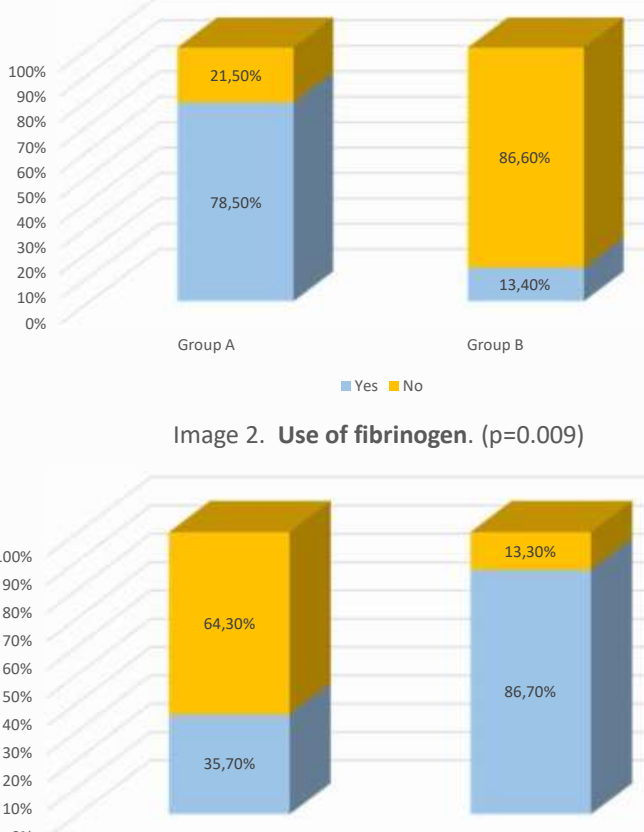

Group A

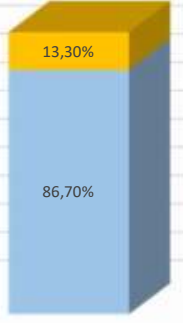

Group B
Image 3. Use of platelets. $(p=0.038)$

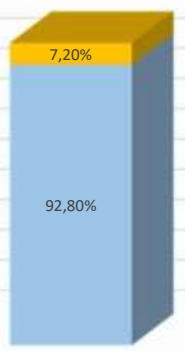

Group A

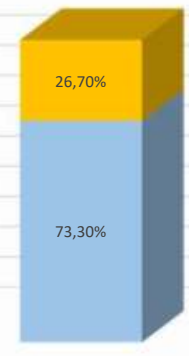

Group B
Image 4. Respiratory complications. ( $p=0.08$ not statistically significant)

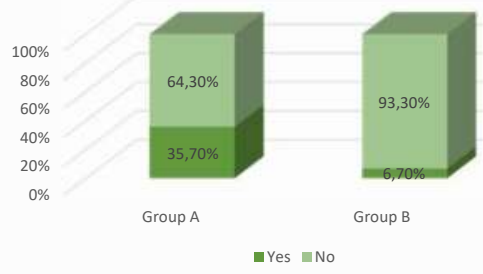

\section{CONCLUSIONS:}

Based on our experience, the use of a viscoelastic tests such as thromboelastometry, allows a substantial change in the management of blood products, with a decrease in the use of fresh frozen plasma and increase in the administration of fibrinogen. Regarding postoperative respiratory complications it seems that increasing our sample could yield to statistically significant results. 